\title{
ANÁLISE DO EFEITO DA VELOCIDADE NO ESCOAMENTO BIFÁSICO EM DUTOS CURVADOS COM VAZAMENTO
}

\author{
L.R.B. SARMENTO ${ }^{1}$, G.H.S. PEREIRA FILHO ${ }^{2}$, E.S. BARBOSA ${ }^{3}$, S.R. de FARIAS NETO ${ }^{4}$ e A.B. \\ de LIMA $^{5}$ (Times New Roman 12) \\ ${ }^{1,4}$ Universidade Federal de Campina Grande, Departamento de Engenharia Química \\ 2,3,5 Universidade Federal de Campina Grande, Departamento de Engenharia Mecânica \\ E-mail para contato: ligiarafaely@ hotmail.com
}

RESUMO - O transporte de petróleo e seus derivados é realizado, na maioria das situações, por meio de dutos interligando os poços de produção em terra, plataformas, refinarias, terminais marítimos e os centros de estocagem. Grandes investimentos têm sido realizados no desenvolvimento de tecnologias para o transporte de fluidos em dutos, visando o aumento do nível de segurança e eficiência de operação Apesar de ser considerado o meio mais econômico de transporte quando comparado às vias ferroviária, marítima e rodoviária, a operação com dutos está sujeita a falhas, ocasionadas pela ação dos agentes presentes na natureza que podem propiciar a deterioração de parte da tubulação, caso não sejam tomadas ações preventivas, tornando as instalações susceptíveis a acidentes operacionais. Sendo assim, o vazamento de um fluido ao meio externo figura como um dos problemas mais sérios na indústria de petróleo, acarretando prejuízos ecológicos e financeiros. O vazamento pode apresentar diversas manifestações de sinais característicos, variação da queda de pressão na posição do vazamento, entre outros parâmetros. As técnicas de detecção de vazamentos disponíveis vão desde a observação visual até a modelagem matemática através de técnicas computacionais. Neste sentido, o presente trabalho propõe investigar numericamente o escoamento bifásico, de uma corrente de óleo, contendo água, em oleodutos com conexões curvadas na presença de vazamentos. Todas as simulações foram realizadas utilizando o pacote comercial Ansys CFX adotando o modelo de partícula para descrever o escoamento bifásico óleo-água e adotando o modelo de turbulência SST em regime transiente. Os resultados das simulações demonstraram que a velocidade de entrada da mistura multifásica exerce influência sobre a queda de pressão no duto na presença do vazamento, onde percebeu-se a existência de uma relação direta entre o aumento da velocidade e a queda de pressão no duto devido ao vazamento.

\section{INTRODUÇÃO}

O transporte de petróleo $\mathrm{O}$ transporte de petróleo e seus derivados são comumente realizados por meio de redes de dutos interligando os poços de produção em terra, plataformas, refinarias, terminais marítimos, parques de estocagem e os centros consumidores. Os dutos representam uma forma de transporte que é considerada mais econômica quando comparado com outras vias, marítima, 


\section{9 a 22 de outubro de 2014 \\ Florianópolis/SC}

ferroviária e marítima, para transportar fluidos (gás ou líquido) por longas distancias (SOUSA, 2007; BEZERRA, 2008; AGUIAR, 2010). Entretanto, diante do crescimento das atividades de exploração e produção, inevitavelmente aumenta-se o risco de eventos acidentais com derramamento de óleo. Inúmeros acidentes vem ocorrendo durante o transporte do petróleo e seus derivados, como por exemplo o vazamento em um oleoduto da Petrobras, na Serra do Mar, que liga a Refinaria Presidente Getúlio Vargas (REPAR), em Araucária, no, demonstrando que as atividades relacionadas ao transporte de petróleo e seus derivados possuem potencial de causar uma variedade de impactos sobre o meio ambiente. Contudo, o número de vazamentos, bem como a quantidade de óleo despejado, pode ser reduzido através de políticas de segurança adotadas pelas empresas encarregadas do transporte.

Existe atualmente uma variedade de técnicas disponíveis para a detecção do vazamento, variando desde a simples inspeção física até os métodos acústicos. Os fatores que vem sendo mais desenvolvidos nessas técnicas são a confiabilidade, eficiência na inspeção dos dutos e rapidez em detectar com precisão possíveis vazamentos ao longo dos desses dutos.

A detecção rápida e precisa de um vazamento possibilita a parada de bombas e fechamento rápido de válvulas impedindo, assim, prejuízos em termos de manutenção e de consumo de energia quer pela indústria petrolífera ou em outros segmentos. Os desastres relacionados com vazamentos de óleo são responsáveis por gastos de milhões de reais de remediação, além de degradarem o meio ambiente. Portanto, o tempo de detecção de vazamento é um parâmetro essencial (COLOMBO e KARNEY, 2002; COLOMBAROLI, 2008).

Desta forma, o conhecimento prévio de todas as variáveis envolvidas no fenômeno de vazamento é crucial para o desenvolvimento de uma técnica eficaz e segura. Porém, na prática muitas dessas variáveis não são facilmente mensuráveis e a fluidodinâmica computacional (CFD) surge como uma ferramenta que auxilia no conhecimento dessas variáveis.

$\mathrm{Na}$ literatura existem poucos trabalhos, utilizando a fluidodinâmica computacional, que tratem da temática de vazamento levando em conta o caráter multifásico e estrutural desses escoamentos. Diante desse cenário, a proposta desse trabalho é realizar uma análise detalhada em torno de um pequeno vazamento em uma tubulação utilizando fluidodinâmica computacional com velocidades típicas e pressão da linha das tubulações. Este estudo é necessário para avaliar a viabilidade de transdução de pressão direta para a detecção de vazamentos e localização

\section{METODOLOGIA}

O problema físico avaliado neste estudo consiste em um fluxo de duas fases, óleo e água, em um duto com 4 metros de comprimento nos ramais horizontal e vertical munido de uma conexão curvada do tipo joelho, com diâmetro de $10 \mathrm{~cm}$ e com um vazamento de forma circular de diâmetro de $4 \mathrm{~mm}$ situado próxima a região da curvatura. A Figura 1 ilustra a geometria do problema tratado neste trabalho e seus detalhes que auxiliam no melhor entendimento das condições de contorno.

O software de fluidodinâmica computacional utilizado nas simulações numéricas conduzidas no presente trabalho foi o Ansys CFX 12.1 e a construção das geometrias e malhas foram construídas 
utilizando o ICEM CFD, ambos constituintes do grupo de software ANSYS 12.1 da ANSYS Technology. A malha, utilizada nas simulações, constituída de 589.745 elementos tetraédricos. Esta malha é o resultado de diversos refinamentos visando obter os melhores resultados possíveis com menor dependência dos resultados numéricos com a malha usada nas simulações, assim como um custo computacional adequado.

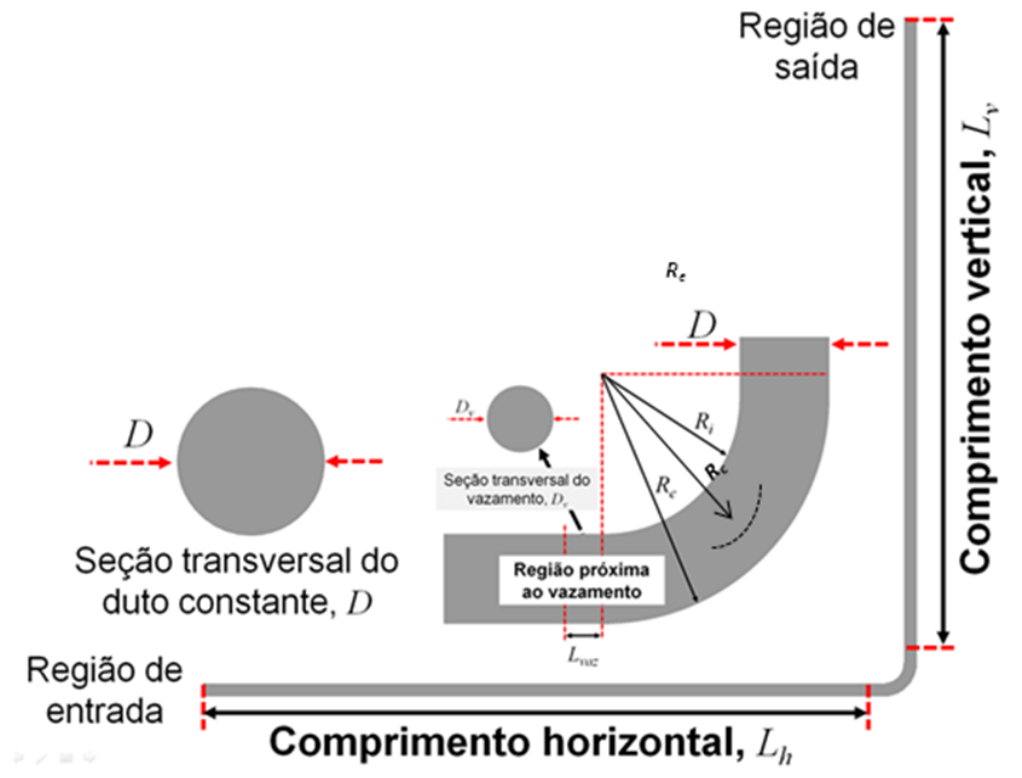

Figura 1 - Geometria da tubulação com a região do vazamento

Modelagem Matemática - Para descrever a hidrodinâmica do escoamento multifásico, as seguintes equações foram utilizadas no presente trabalho:

- Equação da conservação de massa

$$
\frac{\partial}{\partial t}\left(r_{\alpha} \rho_{\alpha}\right)+\nabla \bullet\left(r_{\alpha} \rho_{\alpha} \vec{U}_{\alpha}\right)=0
$$

- Equação de conservação de momento linear

$$
\frac{\partial}{\partial t}\left(r_{\alpha} \rho_{\alpha} \vec{U}_{\alpha}\right)+\nabla \bullet\left[r_{\alpha}\left(\rho_{\alpha} \vec{U}_{\alpha} \otimes \vec{U}_{\alpha}\right)\right]=-r_{\alpha} \nabla p_{\alpha}+\nabla \bullet\left\{r_{\alpha} \mu_{\alpha}\left[\nabla \vec{U}_{\alpha}+\left(\nabla \vec{U}_{\alpha}\right)^{T}\right]\right\}+\vec{S}_{M \alpha}+\vec{M}_{\alpha}
$$

Foi utilizado o modelo de turbulência de duas equações, o SST para a fase contínua (óleo), que tem sua formulação baseada nos modelos $k-\varepsilon$ e $k-\omega$. A proposta do modelo é fazer uma transição suave na maneira do cálculo da turbulência, dependendo da região onde ocorre o escoamento: longe da parede, utiliza-se o k-e, próximo à parede o $k-\omega$. 
Condições de Contorno - As condições de contorno adotadas sobre as fronteiras da geometria em estudo (Figura 1) estão descritas na Tabela 1:

Tabela 1 - Resumo das condições de contorno adotadas para o problema em estudo

\begin{tabular}{c|c}
\hline Seção de entrado da tubulação & $\begin{array}{r}\text { Valor prescrito e não nulo para a componente de velocidade } \\
\text { axial, } \mathrm{U}_{1}=3,0 \mathrm{~m} / \mathrm{s}, \mathrm{U}_{2}=3,5 \mathrm{~m} / \mathrm{s} \text { e } \mathrm{U}_{3}=4,0 \mathrm{~m} / \mathrm{s}\end{array}$ \\
\hline Seção de saída & Valor prescrito para a pressão estatística: $\mathrm{P}=1 \mathrm{bar}$ \\
\hline Seção do orifício de vazamento & Condição prescrita de pressão: $\mathrm{P}=1 \mathrm{bar}$ \\
\hline Rugosidade na parede do tubo & Tubo Liso \\
\hline
\end{tabular}

As propriedades físico-químicas dos fluidos, óleo e água, a 293,15 K usadas nas simulações são mostradas na Tabela 2.

Tabela 2 - Propriedades físico-químicas dos fluidos

\begin{tabular}{c|c|c}
\hline Propriedades Físicas & Óleo (Fase contínua) & Água (Fase dispersa) \\
\hline Densidade $\left(\mathrm{kg} / \mathrm{m}^{3}\right)$ & 860 & 998 \\
\hline Viscosidade dinâmica (Pa.s) & 0,044 & 0,001 \\
\hline Diâmetro da partícula ( $\square \mathrm{m})$ & & 0,001 \\
\hline & \multicolumn{2}{|c}{ Óleo/Água } \\
\hline Tensão Superficial $(\mathrm{N} / \mathrm{m})$ & 0,031 \\
\hline
\end{tabular}

Condição inicial - As simulações realizadas neste trabalho foram feitas em duas etapas. $\mathrm{Na}$ primeira etapa simulou-se o escoamento em regime permanente em um tubo sem orifício de vazamento, ou seja, com a condição de contorno de parede impermeável nessa região e, desta forma, considerando o vazamento desativado. Na segunda etapa, simulou-se o escoamento em regime transiente com a condição de abertura na região do orifício de vazamento e, desta forma, considerando o vazamento ativado. Nesta segunda etapa, utilizaram-se os resultados numéricos da primeira etapa (caso permanente) como condição inicial de simulação do caso transiente. As simulações foram realizadas com o passo de tempo de $0,01 \mathrm{~s}$.

\section{METODOLOGIA}

A avaliação da queda de pressão devido à presença de vazamento no duto foi feita tomando-se dois planos perpendiculares ao eixo central do mesmo (Figura 2), um a montante do vazamento (distante $2 \mathrm{~m}$ antes do orifício, no ramal horizontal) e outro a jusante do vazamento (distante $2,0 \mathrm{~m}$ 
depois do orifício, no ramal vertical). Tomou-se a média ponderada da pressão total em cada plano, onde no Ansys $\mathrm{CFX}^{\circledR}$ este valor é calculado levando-se em consideração todos os elementos da malha, sem favorecer regiões de maior densidade de elementos ou pontos nodais da malha nesta seção. A região definida para análise é chamada de trecho

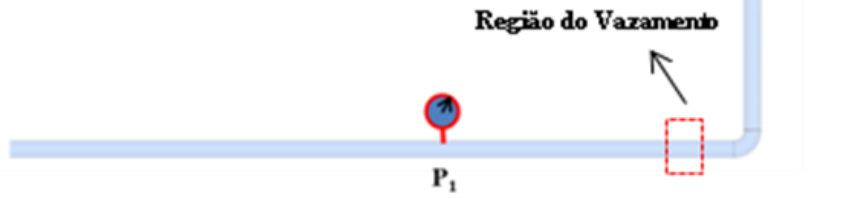

Figura 2 - Trecho utilizado para análise da queda de pressão

A Figura 3 apresenta a evolução da queda de pressão com o tempo no trecho avaliado, conforme ilustrado na Figura 2, referentes aos Casos estudados.

O comportamento para os casos com velocidade de entrada, $3 \mathrm{~m} / \mathrm{s}, 3,5 \mathrm{~m} / \mathrm{s}$ e 4,0 m/s sobre a queda de pressão no duto na presença de vazamento foi observado que a queda de pressão nos primeiros $0,001 \mathrm{~s}$ se mantém praticamente constante, período em que não há vazamento. Houve uma pequena variação da pressão, aproximadamente 0,01 bar para todos os casos.

Para um tempo superior a 0,001s foi definida a condição de vazamento de pressão prescrita, 1 bar. Foi observada uma perturbação na curva (Figura 3) no período entre 0,001e 0,002 s e, a partir deste momento, observa-se um decréscimo na queda de pressão até atingir um valor praticamente constante indicando, assim, que o escoamento atingiu o equilíbrio com a presença do vazamento. Isso pode ser explicado pelo diâmetro do furo que é muito pequeno. 


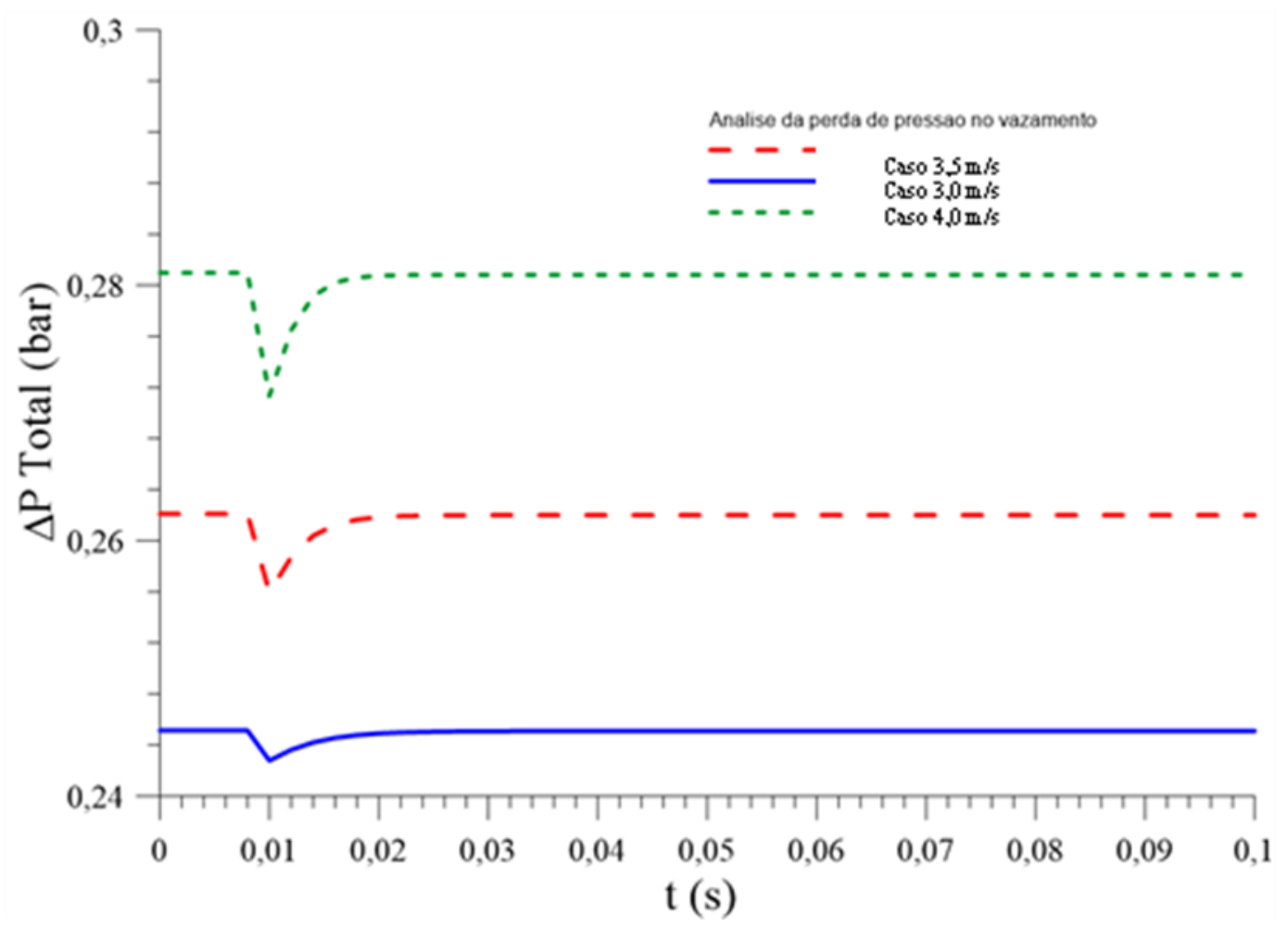

Figura 3 - Comportamento da variação de pressão total no trecho considerado em função do tempo para o orifício de vazamento no tubo - Casos 08, 10 e 11.

Os resultados demonstrados apontam que a velocidade de entrada da mistura multifásica exerce influencia sobre a queda de pressão no duto na presença do vazamento, onde foi percebido que à medida que a velocidade de entrada aumenta a queda de pressão também aumenta.

A Figura 4 apresenta o comportamento da evolução da pressão com o tempo a montante e a jusante do ponto de vazamento. Os resultados mostram um comportamento análogo ao obtido para a queda de pressão no trecho analisado na Figura 3, que pode ser visto na Figura 4, porém com ordem de grandezas. 


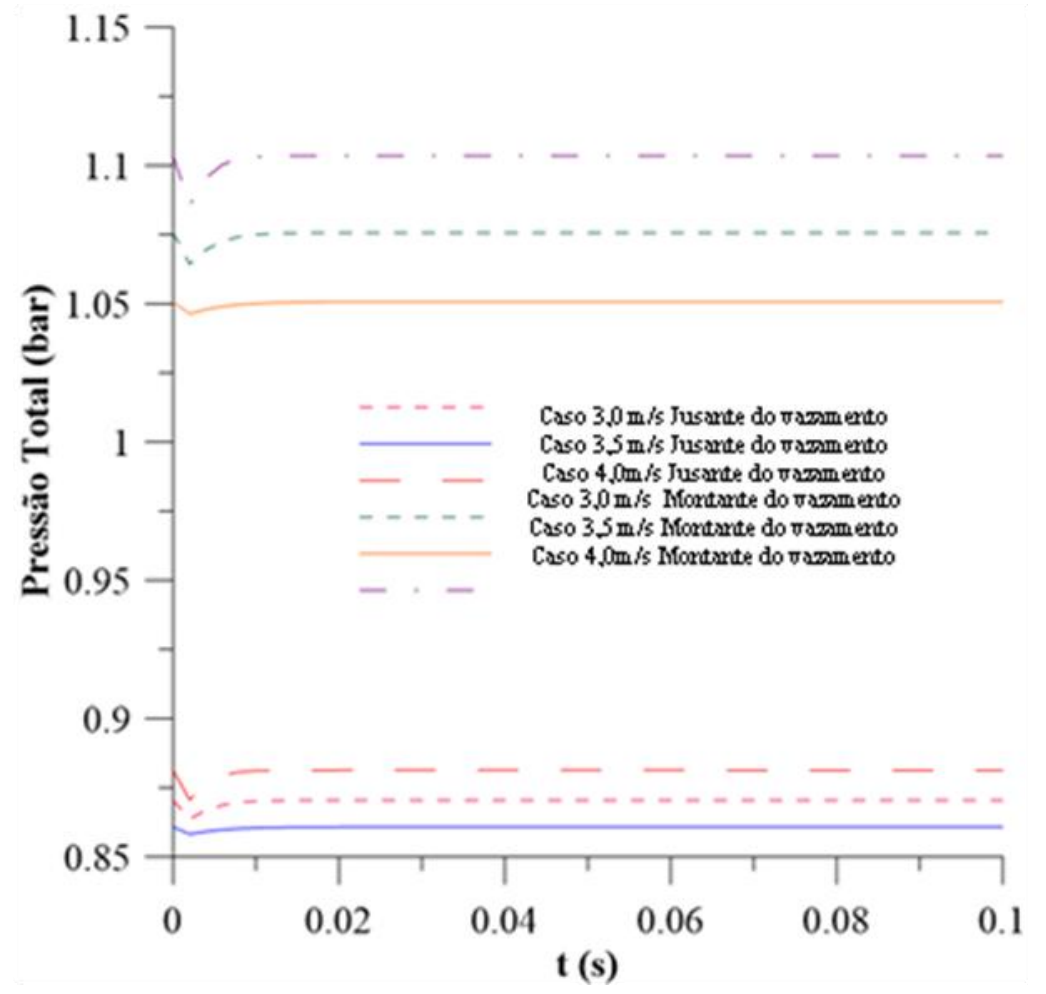

Figura 4 - Comportamento da pressão total em função do tempo analisado em um plano $2 \mathrm{~m}$ a montante do vazamento e em um plano $2 \mathrm{~m}$ a jusante do vazamento para os Casos estudados

\section{CONCLUSÕES}

Através dos resultados obtidos foi possível identificar características do fenômeno vazamento. Observou-se através dos resultados numéricos das simulações de escoamento bifásico que quanto maior a velocidade de escoamento, maior será a perturbação causada por um vazamento.

\section{AGRADECIMENTOS}

Os autores gostariam de expressar seus agradecimentos às agências brasileiras PETROBRAS/UFCGANP-25, CNPq, CAPES, FINEP e JBR Engenharia Ltda. por apoiar este.

\section{REFERÊNCIAS}

AGUIAR, F. G. Utilização de Redes Neurais Artificiais para Detecção de Padrões de Vazamentos em Oleodutos. 2010. 95 p. Dissertação (Mestrado em Engenharia Mecânica) Universidade de São Paulo, Escola de Engenharia de São Carlos, São Paulo, 2010. 
AZEVEDO, F. M. Proposta de Algoritmo para Detecção de Vazamentos em Oleodutos Utilizando Análise Frequencial de Sinais de Pressão. 2009. 105 f. Dissertação (Mestrado em Engenharia Elétrica) - Universidade Federal do Rio Grande do Norte, Centro de Tecnologia, Natal, RN, 2009.

BEZERRA, B. A. F. Detecção de vazamentos em tubulações de gás pelo método de transitório de pressão utilizando CLP e sensores. 2008. 77 f. Monografia. (Especialização em Engenharia de Instrumentação) - Universidade Federal de Pernambuco, Recife, PE, 2008.

COlOMbaroli, P. L. S. Sistema de Detecção de Vazamento em Dutos de Petróleo. Universidade Federal de Itajubá, Itajubá. Disponível em: <www.anp.gov.br/site/extras/prh/docs/ANP_10anos/PRH_16.pdf> Acesso em: 24 fev. 2010.

COLOMBO, A. F., KARNEY, B. W. Energy and Costs of Leaks: Toward a Comprehensive Picture. Journal of Water Resources Planning and Management, ASCE, vol. 128, n. 6, p. 441-450, 2002. BEN-MANSOUR, R., HABIB, M. A., KHALIFA, A., YOUCEF-TOUMI, K., CHATZIGEORGIOU, G., Computacional Fluid Dynamic Simulation of Small Leaks in Water Pipelines. Computers \& Fluids, 2012 .

SOUSA, E. O. Detecção de Vazamento em Tubulações Através do Método Acústico e da Análise de Transiente de Pressão. 2007. 160 f. Dissertação (Mestrado em Engenharia Química) Universidade Estadual de Campinas, Faculdade de Engenharia Química, Campinas, SP, 2007. 